\title{
Content-Based Retrieval of Retinal Images for Maculopathy
}

\author{
K Sai Deepak \\ CVIT, IIIT Hyderabad \\ AP, India, 500032 \\ sai.deepak@ research.iiit.ac.in
}

\author{
Gopal Datt Joshi \\ CVIT, IIIT Hyderabad \\ AP, India, 500032 \\ gopal@ research.iiit.ac.in
}

\author{
Jayanthi Sivaswamy \\ CVIT, IIIT Hyderabad \\ AP, India, 500032 \\ jsivaswamy@iiit.ac.in
}

\begin{abstract}
A growing number of public initiatives for screening the population for retinal disorders along with widespread availability of digital fundus (retina) cameras is leading to large accumulation of color fundus images. The ability to retrieve images based on pathologic state is a powerful functionality that has wide applications in evidence-based medicine, automated computer assisted diagnosis and in training ophthalmologists. In this paper, we propose a new methodology for content-based retrieval of retinal images showing symptoms of maculopathy. Taking the view of a disease region as one which exhibits deviation from the normal image background, a model for the image background is learnt and used to extract disease-affected image regions. These are then analysed to assess the severity level of maculopathy. Symmetry-based descriptor is derived for the macula region and employed for retrieval of images according to severity of maculopathy. The proposed approach has been tested on a publicly available dataset. The results show that background learning is successful as images with or no maculopathy are detected with a mean precision of 0.98 . An aggregate precision of 0.89 is achieved for retrieval of images at three severity-levels of macular edema, demonstrating the potential offered by the proposed disease-based retrieval system.
\end{abstract}

\section{Categories and Subject Descriptors}

H.3 [Information Storage and Retrieval]: information search and retrieval, content analysis and indexing

\section{General Terms}

Algorithms

\section{Keywords}

Diabetic retinopathy, Image background learning, Image retrieval, Maculopathy, Retinal image

Permission to make digital or hard copies of all or part of this work for personal or classroom use is granted without fee provided that copies are not made or distributed for profit or commercial advantage and that copies bear this notice and the full citation on the first page. To copy otherwise, to republish, to post on servers or to redistribute to lists, requires prior specific permission and/or a fee.

IHI'10, November 11-12, 2010, Arlington, Virginia, USA.

Copyright 2010 ACM 978-1-4503-0030-8/10/11 ...\$10.00.

\section{INTRODUCTION}

Diabetic Retinopathy (DR) is a complication of the retina due to diabetes and is a leading cause of blindness in urban population. Early diagnosis through regular screening and treatment has been shown to prevent visual loss and blindness. Digital color fundus photography allows acquisition of fundus (retina) images in a noninvasive manner which makes large scale screening easier. Recently, there has been significant effort towards building screening solutions for DR [5] using color fundus images (CFI) mainly due to the value they offer such as low-cost and wider reach.

Increase in the awareness of DR followed by initiatives like large scale public screening programmes have lead to collection of large number of CFI even in a single day. Due to the shortage of sufficient retina experts at the examination centers, an initial screening is performed by a "Reader" who is trained to analyze these CFIs and report the presence of lesions and/or stage the disease of interest. Correct evaluation of these images is an essential step during the initial screening. However, image variations arising from difference in eye pigmentation among population, light reflection artifacts etc pose challenges. Furthermore, accuracy of evaluation significantly varies with the level of experience of the "Reader".

A solution to the above problems is to capitalize on the volume of data collected and previously analyzed. Specifically, an image repository formed over a period of time with /without diagnosis information can be a valuable asset as it captures a range of image variations within a disease profile and has the potential of aiding evidence-based examination. A retrieval system built on top of such a repository can assist in evaluation of CFIs by retrieving similar images from image repository and make available associated diagnostic information or aid through automatic suggestion. Such systems can significantly improve and accelerate the evidencebased learning process via which a "Reader" (or medical student) usually acquires during training. In this paper, our focus is on developing disease-specific image retrieval system for retinal images to meet these objectives.

Retinal exams for DR follow a well defined protocol where the "Reader" looks for specific visual signs of the disease. In CFI, DR is mainly characterized by the presence of lesions like hard exudates, hemorrhage or microaneurysms (shown in fig. 1). Maculopathy is an advanced stage of DR in which lesions occur close to the macula, the part of retina that provides clearest vision. It is indicative of macular edema, which is characterized by swelling in macula potentially leading to irreversible blindness. Sight-threatening 

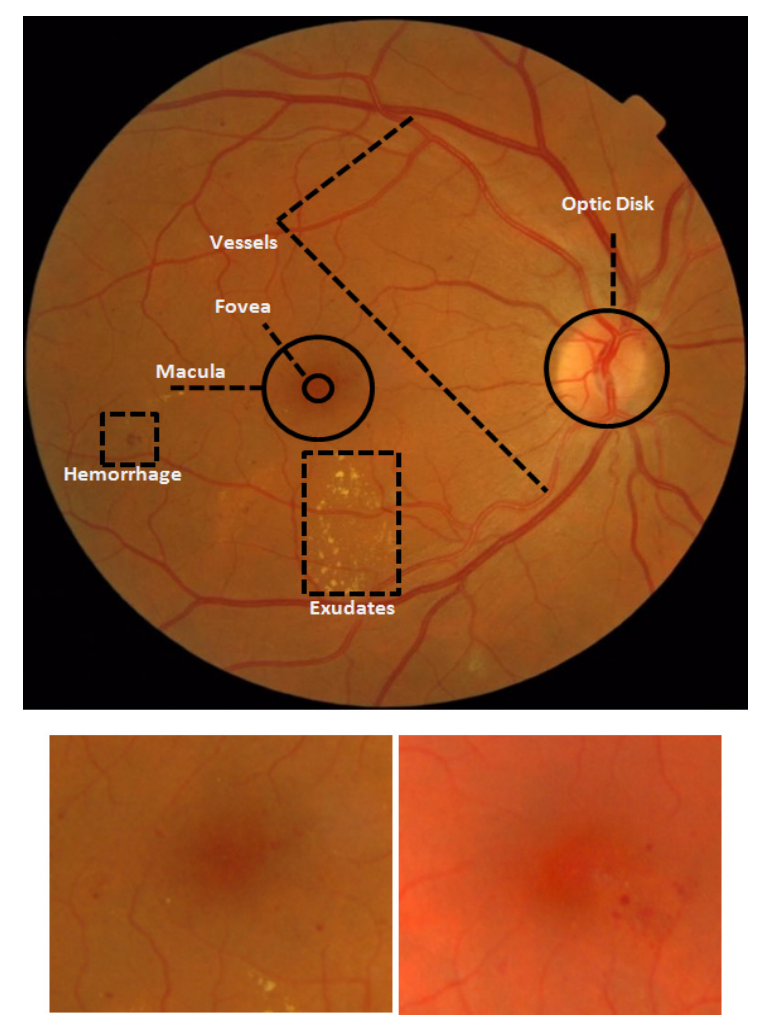

Figure 1: (Top) Color Fundus image with anatomical structures and disease annotated. (Left) Macular Edema with moderate severity. (Right) Macular Edema with high severity.

macular edema always co-occurs with visible signs of retinopathy in the macula region [16]. Therefore severity-level of maculopathy is used to assess the risk of clinically significant macular edema. The severity level is based on the distance of the DR lesions from the centre of the macula, with presence of lesions within 1 papilla diameter deemed as severe. This paper addresses maculopathy and presents a method for retrieving images based on similarity between a given image and images with macular edema of different levels of severity available in the repository.

Content Based Image Retrieval (CBIR) based solutions have been explored for developing medical imaging solutions. An early attempt for retinal images finds global and local features based on color, texture, composition and structure and retrieval is achieved by applying a standard similarity metric [7]. This only retrieves similar looking retinal images regardless of the underlying disease. Towards this end, a Bayesian framework with CBIR has been explored for diagnosis using a large database. Statistical features of a DR lesion containing regions are mapped on to a semantic space corresponding to disease states in $[18,17]$ using Fischer discriminant. This is followed by retrieval of similar images using an L-norm distance between the query image and archived image descriptors in the mapped space. Diagnosis of DR with categorization to different disease stages is then addressed using a simple k-nearest neighbor based voting scheme. Both these systems are semi-automated as they rely on manual segmentation of lesions from images.

Retrieval of multimodal images has been proposed by com- bining wavelet decomposition of images for content signature along with patient related information [14, 13]. These methods rely on global signatures of the images for content similarity and do not attempt to stage a disease.

The method in [1] aims to propose a completely automated disease separation method to assist in CBIR for Stargardt's disease and age related macular degeneration (AMD). Later stages of Stargardt's disease is characterized by appearance of annular atrophy around fovea which is detected by finding circular regions of strong contrast using Gradient Inverse Coefficient of variation statistics. AMD is seen with appearance of drusen which are round white-yellow deposits on retina. An area morphology-based granulometry along with texture features described by the AM-FM model of the image is used for detecting and characterizing drusen. Even though the detection and characterization of these disease shows promise, this system is yet to be implemented within a CBIR framework.

We note that there is an incompleteness in combining automated disease detection with disease specific retrieval of images in existing approaches. Detection of abnormalities, associated with a disease, needs to be automated because it is generally the case that an image repository does not have annotation available for all the images. We aim to design a system which combines automatic detection of abnormal regions in an image and uses the information about this region for disease-based retrieval. In this work, the system design focuses on macular edema and the retrieval is based on severity level which depends on the visual appearance of its manifestations and their location. The proposed system is novel in two aspects: a) automatic detection of the abnormal regions is done via learning the normal background (non-vessel regions) rather than learning the lesion patterns (hard exudates, hemmorhages and microaneurysms) as in previous approaches and b) the retrieval uses a similarity measure based on the polar asymmetry around the macula.

\section{PROPOSED METHOD}

In a typical CBIR system, image descriptors are first indexed into a query database for large number of images, in an offline process, followed by querying the database online with a new query image [10]. Hence, the entire workflow for the retrieval system we propose is divided into three steps: learn the background from normal images, compute image descriptors to build a query database and retrieve similar images from the query database based on the presence or absence of pathology around the macula. Learning the normal image background is modeled as a single-class learning problem. Here, background consists of non-vessel regions around the macula. We have used the Messidor ${ }^{1}$ dataset [8] for training and validating our system. (sample images shown in fig. 1).

The workflow of the offline process consists of two separate set of steps, a) learning background to build the normal subspace from a database of normal CFI's (see left branch in fig. 2) and b) indexing new images into the "Query Database" by computing their descriptors after disease patches are (see right branch in fig. 2). Preprocessing and extracting patch features are common steps among these two steps. Preprocessing includes the following: extraction and enhancement

\footnotetext{
${ }^{1}$ Kindly provided by the Messidor program partners (see http://messidor.crihan.fr)
} 


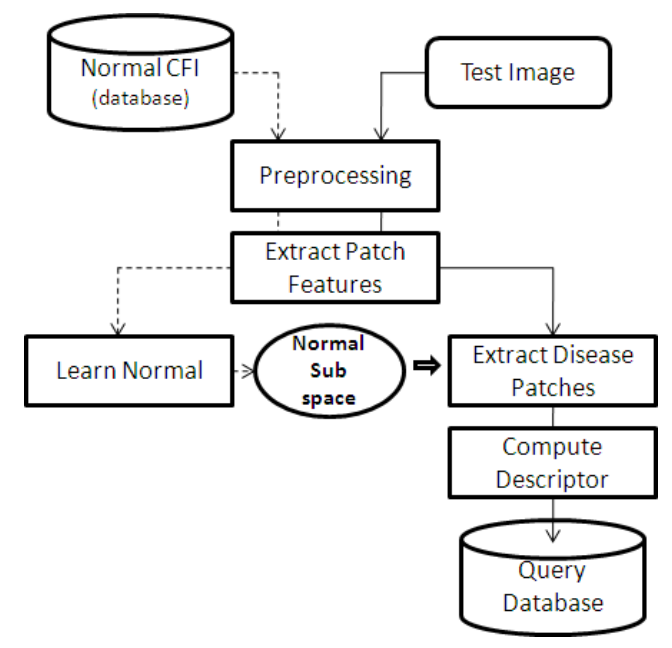

Figure 2: Workflow for building disease image database. The left hand branch shows the flow for learning background. The right hand side corresponds to indexing the query database.

of a region of interest (ROI), namely the macula which is defined as 1 papilla diameter from the fovea and vessel removal.

A circular region of diameter equivalent of 2 papilla diameter around the macula is considered as the desired ROI for further processing. A bias field removal step followed by color enhancement of the interest region is performed. Blood vessels are not of interest for this work; therefore they are detected and removed. From the remaining regions, fixed-size patches are extracted and for each patch, intensity histograms from scale space and histogram of local binary patterns are computed as descriptors for background learning. Single class learning with k-means data description is used for this purpose.

Next a "Query Database" consisting of image descriptors for similarity based retrieval is created for a new set of images. A new image is again preprocessed as described earlier. All non-vessel patches in the ROI of the new image are marked as normal or abnormal based on the knowledge of normal sub-space. The abnormal regions in the image are given a large weight for further processing. A descriptor estimating the polar asymmetry of macula is computed for this image and indexed into the "Query Database". This entire process is performed offline.

Querying an image database for content based retrieval is an online process (see fig. 3). A query image is preprocessed as described earlier and a polar asymmetry descriptor is computed. This descriptor is then compared with the indexed images in the query database using an L2-norm metric for retrieving similar images. Performance of the retrieval operation is measured in terms of precision and accuracy. Rest of the paper is organized as follows. Section 3 describes the process depicted in fig. 2 in detail. Section 4 introduces a new descriptor based on polar asymmetry of the macular for image retrieval. Section 5 shows the results for image retrieval with a discussion on the negative results obtained for one of the disease classes. This is followed by conclusion and future work in section 6 .

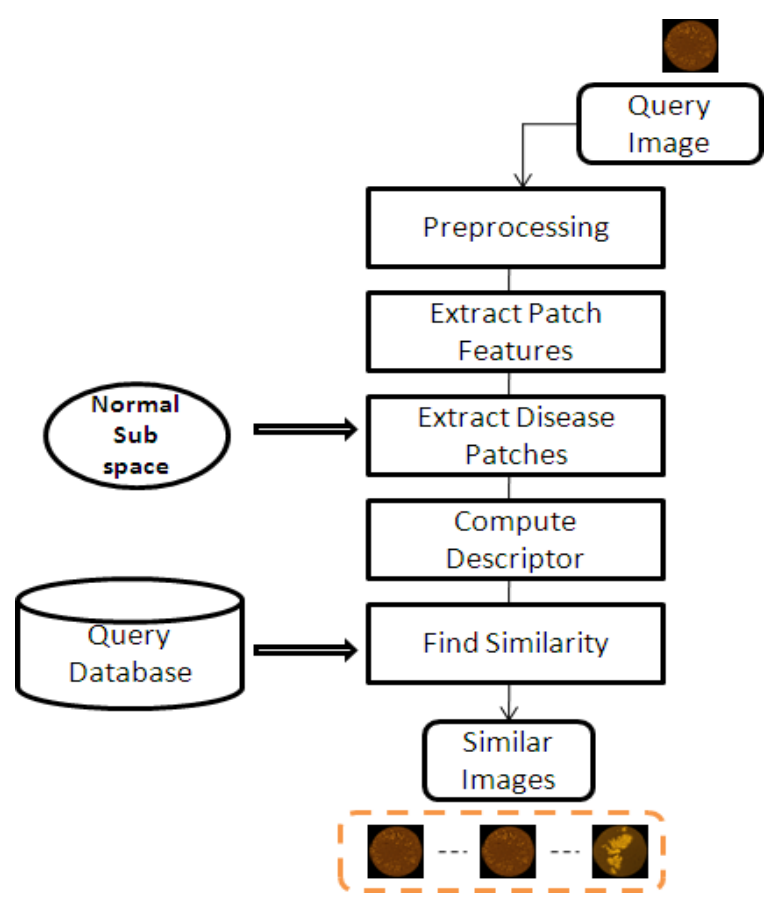

Figure 3: Workflow for on-line content based image retrieval.

\section{LEARNING NORMAL BACKGROUND}

\subsection{Preprocessing}

Retinal images are plagued by imaging artifacts. The most common among them is the introduction of illumination bias in CFI due to the spherical nature of the retina. This is also known as bias field. Another common problem is the variation in the distribution of pigmentation known as tiger striping which clearly appears as greenish stripes on the red-orange background. One of the aims of preprocessing is to address these problems.

Since the green channel of CFI provides maximum contrast for distinguishing between structures and lesions, the bias field estimation and removal is done in the green channel. Results for bias correction in color space are also presented for qualitative analysis. This is followed by vessel detection.

\subsubsection{Bias Field Removal}

The bias field is estimated as a multiplicative, slow-varying function over the interest region. Thus, an image is modeled as:

$$
Y_{k}=X_{k} G_{k}
$$

where, $Y_{k}$ is the observed image, $X_{k}$ is the actual radiance, $G_{k}$ is the bias introduced in the image and $k$ is the position vector.

In logarithmic form equation (1) can be written as,

$$
y_{k}=x_{k}+g_{k}
$$

The bias field is estimated to compensate for intensity inhomogeneities using modified fuzzy c-means objective function [3]. The fuzzy c-means objective function is optimized 


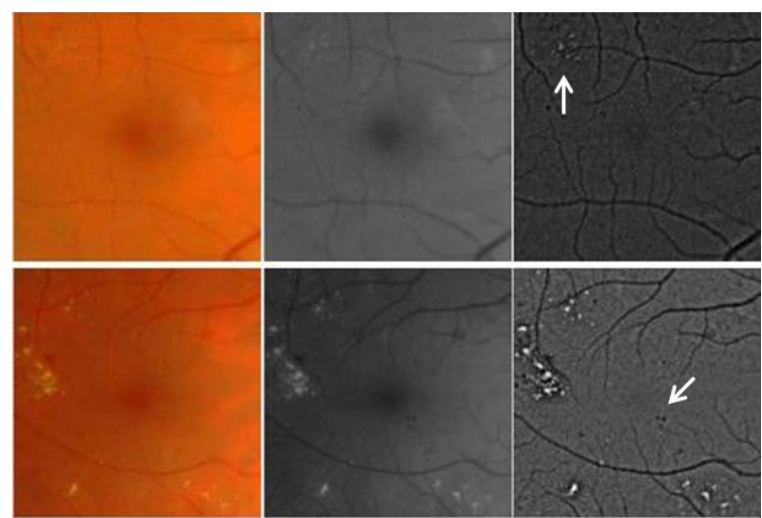

Figure 4: Original versus Bias Corrected green channel image.

to account for the membership of neighboring pixels while estimating the bias field. The bias field $g_{k}$ is computed as,

$$
g_{k}=y_{k}-\frac{\sum_{i=1}^{c} U_{i k} V_{i}}{\sum_{i=1}^{c} U_{i k}}
$$

where, $V_{i}$ is the cluster representative, $U_{i k}$ is the partition matrix and $c$ is the number of clusters.

The original CFI and their green channel components in fig. 4 can be seen to have slow illumination variation from left to right. The image in the bottom row also suffers from tiger striping. As can be observed from the corrected results in the last column, the correction step normalizes most of this variation while enhancing the contrast of lesions. For instance, the bright lesions in the top row and dark lesions in the bottom row (indicated by arrows) have greatly improved visibility in the corrected results whereas they are nearly invisible in the original. Results of bias correction, for the same images, in RGB space can be seen in fig. 5. To improve the color contrast of the resultant image the following operations are applied to the $\mathrm{r}, \mathrm{g}$ and $\mathrm{b}$ color planes.

$$
\bar{r}=\frac{g_{\text {corr }}}{v} * r ; \quad \bar{g}=\frac{g_{\text {corr }}}{v} * g ; \bar{b}=\frac{g_{\text {corr }}}{v} * b
$$

where, $g_{\text {corr }}$ is the corrected green color plane and $\bar{r}, \bar{g} \bar{b}$ are the corrected images for red $r$, green $g$ and blue $b$ color plane respectively. $v$ is the maxima of three color planes $v=\max [r, g, b]$

It must be noted that though the coloring of the corrected images do not represent the natural retinal appearance, the contrast of the background with the lesions has improved which is essential for subsequent processing.

The resulting bias corrected images in color space reaffirm the previous observations made for green color channel images. Other than enhancing the contrast of retinal structures and normalizing pigmentation effect it can also be observed that the bright lesions on the top-left of fig. 5(top row) and dark lesions within macula in fig. 5(bottom row) are enhanced. These lesions are not clearly visible in the original color image.

\subsubsection{Vessel Detection and Removal}

Since the characteristics of blood vessel fragments are similar to those of the dark lesions like hemorrhage and microaneurysms, vessels need to be removed. Blood vessels are first

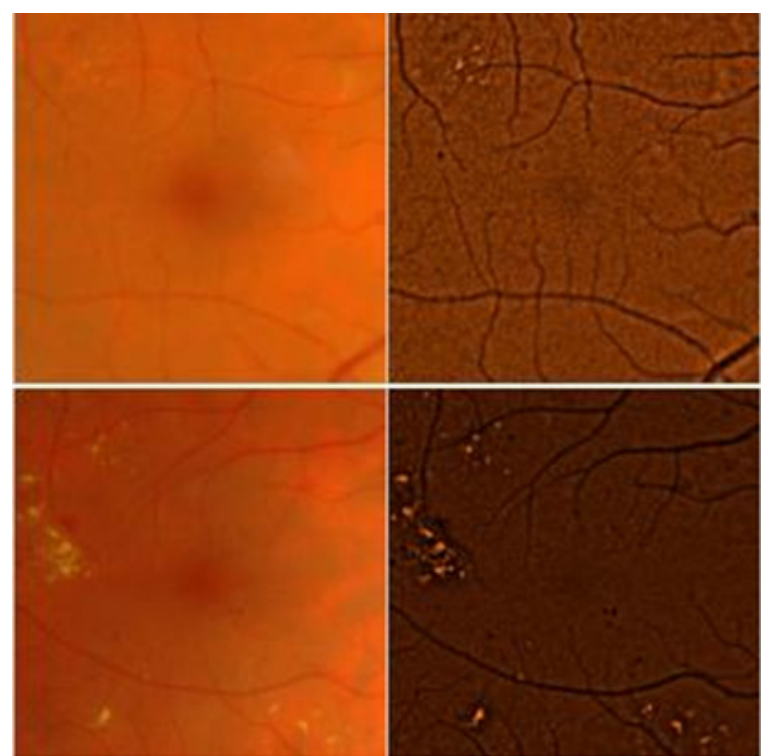

Figure 5: Original versus Bias Corrected rgb color image.

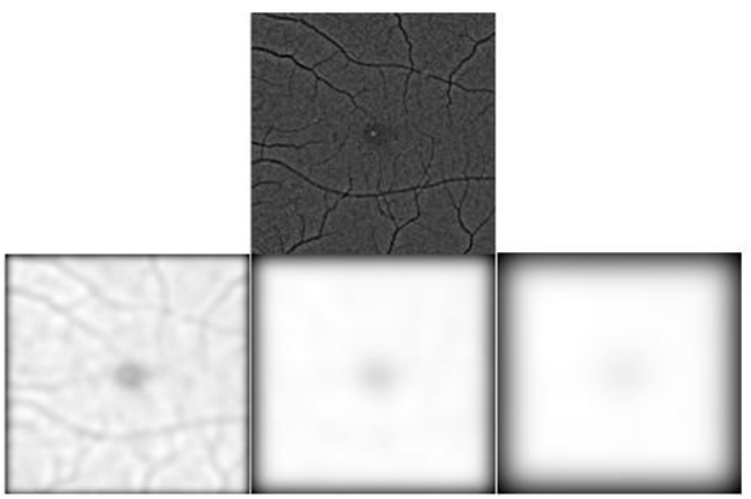

Figure 6: Green channel image of ROI and estimated background with increasing kernel size.

detected from the interest region using the method reported in [6] and they are excluded from further processing.

\subsection{Feature Extraction}

\subsubsection{Intensity Features in Scale Space}

DR manifestations are characterized by nonuniform intensity variation against the retinal image background. Continuous smoothing of image with a large Gaussian kernel gives a good estimate of the image background (shown in fig 6). For an abnormal image, taking the difference of the original image with different background estimates will produce images where the disease patterns (lesions) are enhanced with varying degree of coarseness. We aim to learn the statistical variation of a normal image with its estimated background to differentiate it from the lesions in the abnormal images.

For estimating the background, each image $I($.$) is smoothed$ with a Gaussian kernel of increasing size to obtain a set of level space images $I(. ; \sigma)$. This is sampled to produce desired background estimates $b_{i} ; i=1,2 . . N$ such that in the 


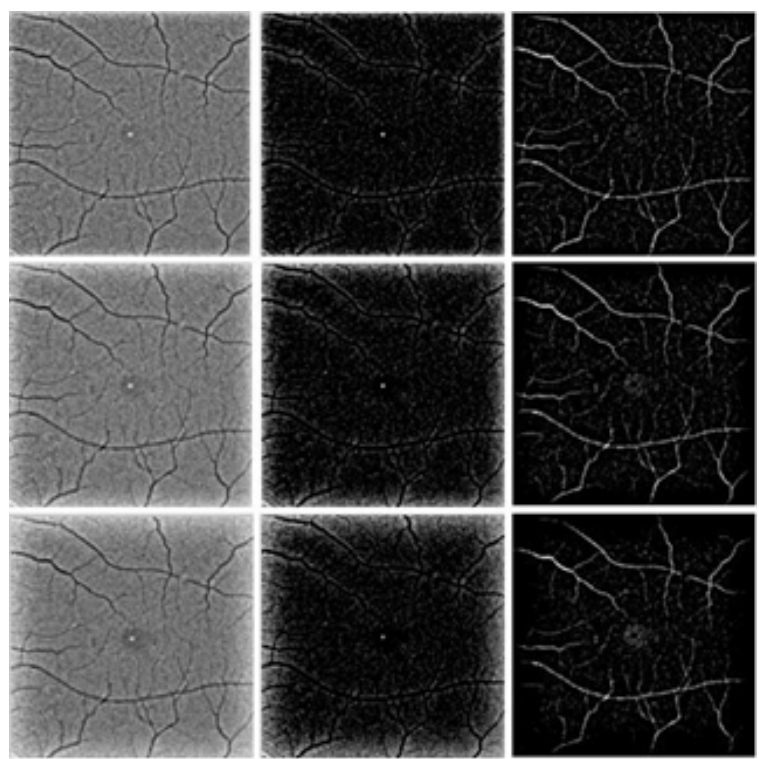

Figure 7: Difference images at increasing scale and the representative bright and dark lesion images.

first level $(i=1)$ the smallest vessels are absent while at the last level $(i=N)$ the largest vessels are absent. Next, a set of difference images $c_{i}=I-b_{i}$ are obtained which are further rectified to derive images $c_{i+}$ and $c_{i-}$ (shown in fig. 7 ). The rectification helps separate darker structures, like vessels from brighter structures. Histograms of $c_{i+}$ and $c_{i-}$ are computed for every non vessel patch and concatenated to construct the desired feature set. These feature set obtained from the green channel of normal images are used for learning the normal sub-space.

Regions with abnormalities will be characterized by higher intensities, than normal regions, in the rectified outputs. This difference will help in separating a normal background patch from a disease patch.

\subsubsection{Texture Features}

The histogram-based feature set presented above captures only part of the information about the normal background. Since the background is actually composed of a dense, fine capillary network it has a characteristic texture. Hence, we derive a second descriptor to capture the same.

Texture features have earlier been used to differentiate among different retinal structures and detection of lesions in diabetic retinopathy [2]. We use local binary patterns (LBP) to describe the surface textures of normal images. LBP effectively represents local spatial pattern and gray scale contrast. It operates on eight neighboring pixels using the center as a threshold. By definition, LBP is invariant to any monotonic transformation of the gray scale and can be computed quickly. Histogram of LBP for each nonvessel patch is computed as a feature set from the normal green channel image. A single feature vector is formed by combining both intensity and texture features.

\subsection{Single Class Learning}

A single class learning problem is a special type of classification problem where all the training data belongs to the target class and data samples differing from the training

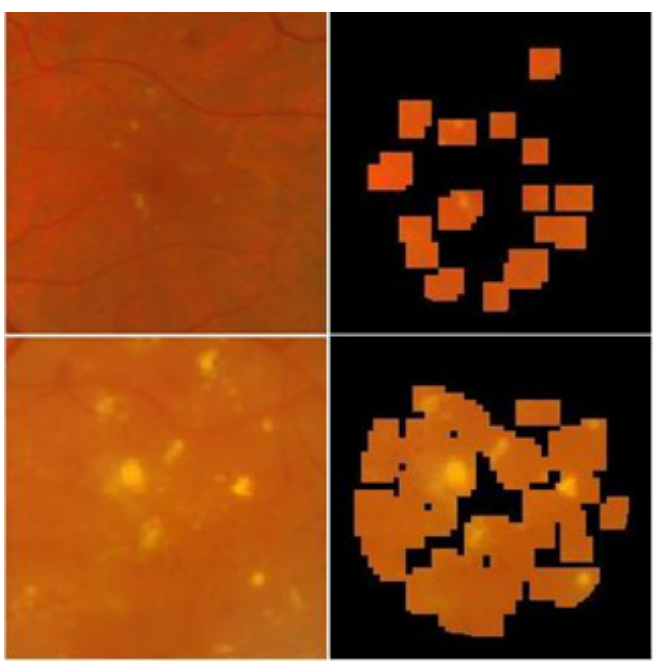

Figure 8: Original image and Corresponding Images marked with disease patches within 'ROI'.

samples belong to the outlier class. Here, the target class is the normal sub-space where feature sets corresponding to fixed size patches from region of interest for normal images are used as training samples. The k-means data description is used for learning the normal sub-space where the target class is described by $\mathrm{k}$ clusters placed such that the average distance to a cluster center is minimized [4]. Therefore the target class is characterized by,

$$
f(x)=\min _{i}\left(x-c_{i}\right)^{2}
$$

where, $c_{i}$ is the $i_{t h}$ cluster centre and $i$ is a real number.

A new image patch falls in the normal sub-space if the minimum distance of its feature vector from all the cluster centers is below a threshold identified experimentally. This patch is considered abnormal otherwise (as example is shown in fig. 8). The details on the number of images used for training the normal sub-space is given in section 5 .

It can be observed from fig. 8 that along with the abnormal patches in the image, some normal patches will also characterized as abnormal. We deal with these falsely marked patches during retrieval by exploiting the polar symmetry of macula.

\section{RETRIEVAL}

Given an image $I_{g}$, to retrieve images $\left[I_{s_{i}} ; i=1,2 . . N\right]$ which are similar to $I_{g}$, a similarity function is required which essentially computes the distance between the features extracted from $I_{g}$ and $I_{s_{i}} ; i=1, \ldots M$ with $N<M$ being the total number of images in the repository. In literature, many distance metrics $[9,11]$ have been used for this purpose. The problem at hand is to retrieve images based on severity level of macular edema. Clinically, severity is decided based on the location of lesions relative to the fovea (at the centre of macula). Hence, the similarity function has to be location-dependent. However, distance to fovea of the lesion cannot be used as a similarity metric as it will be sensitive to the accuracy of the lesion localization. Instead, we propose a novel descriptor which incorporates lesion distance for similarity based on the symmetry around the macula, as 


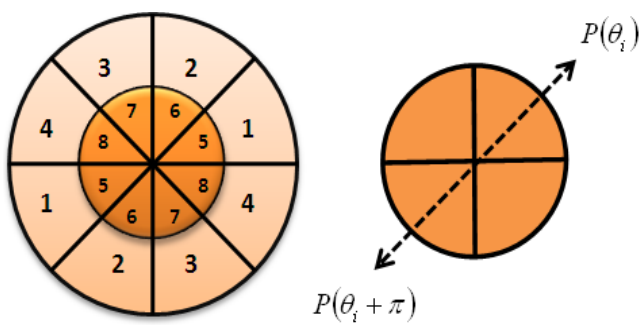

Figure 9: (Left) (a) Representation of polar symmetry in the macula. (Right) (b) Diametrically opposite patches on a polar grid.

symmetry is an important characteristic in medical images. A polar grid is imposed on the ROI which is used in the assessment of the degree of symmetry of the image. The advantage of using a polar grid is that it provides a natural way of analyzing the image in a spatially-variant manner: the assessment area increases with increasing distance from the centre in the polar grid. This is attractive as lesion presence in the central region (signifying highest severity level) can be determined via a finer analysis compared to the peripheral regions. Next, we describe how this symmetry is measured and incorporated in the proposed descriptor.

First, a symmetry measure is defined as the distance between the histograms of diametrically opposite pair of patches. A descriptor is next constructed using a weighted combination of these distances. We illustrate this with an example. Consider the case where the grid is divided into 4 patches of equal size as shown in fig. 9b. The derived descriptor is a vector $v$ defined as

$$
v=\left[a_{j} * d_{j}: j=1,2\right]
$$

Here, $a_{j}$ are binary valued weights, $d_{j}$ is the distance between the histograms of the patches $P_{\theta_{j}}$ and $P_{\theta_{j}}+\pi$. The absence or presence of lesions in a pair of patches is used to determine the weights $a$.

In our implementation, we used 2 radial and 8 angular samples to give 16 patches as shown in fig. 9 (a). The weights $a$ were taken to be $k$ and $100 k$ ( $k$ is a constant) for the cases of absence and presence of lesions, respectively. For a query image $I_{g}$, the similarity to all the images $\left[I_{s_{i}}\right.$; $i=1,2 . . M]$, in the query database is computed for each annulus separately. A coarse comparison is performed between images by computing the L2-norm of the mean of descriptors to differentiate normal from abnormal. The images from the database with least distances are deemed to be most similar to the query image.

This method of finding a symmetry-based descriptor on a polar grid can be further generalized by varying the number of radial/annular sampling and the orientation of patches being compared.

\section{EXPERIMENTAL RESULTS}

The proposed system was constructed and tested using the Messidor database [8]. The database consists of CFI captured at 8-bits per color plane, and of size 1440 x 960 or $2240 \times 1488$ pixels. These images are acquired with or without pupil dilation. All the images are annotated by experts with three severity levels for macular edema. The total number images is 342 of which 205, 41 and 96 are the numbers
Table 1: Retrieval results for Normal, Moderate and Severe classes of macular edema.

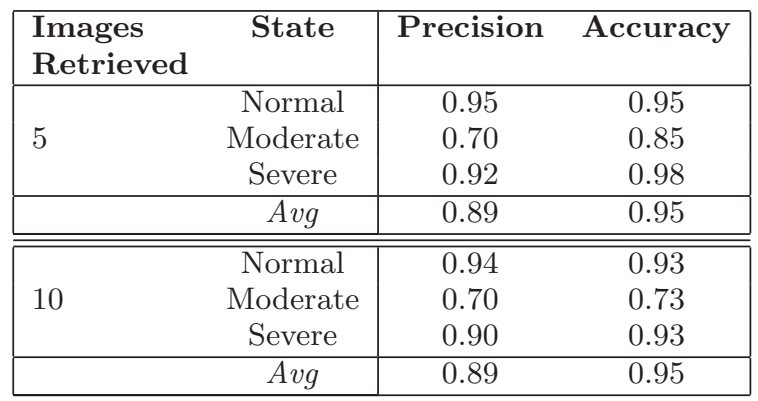

of images at severity levels 0 (normal), 1 (moderate) and 2 (severe).

Automatic macula detection from CFI images has been studied and several solutions have been proposed [15, 12]. Since it is not the focus of this work, we extracted the center of macula manually for all images. For the purpose of learning the normal sub-space, 90 images with severity level 0 are used. Remaining 252 images are used for testing the CBIR workflow. Two metrics are used to measure the retrieval performance at the severity-level, precision and accuracy. Precision is defined as,

$$
\text { precision }=\frac{(\text { relevant } \text { images }) \cap(\text { retrieved images })}{\text { retrieved images }}
$$

Average precision is computed for all 252 test images for the top 5 and top 10 similar images retrieved (shown in table. 1, 2). Since the number of images in each class is always more than the number of images retrieved, the recall rate is not computed.

Accuracy of classifying a query image to its correct severity class based on top $\mathrm{k}$ results was assessed as follows. If the maximum of number of the $\mathrm{k}$ images retrieved for a query image belongs to the correct severity class then the accuracy of classification for that image is taken to be 100 percent otherwise zero. Average accuracy was computed for all the test images as defined in Eq. 8.

$$
\text { accuracy }=\frac{\text { images classified correctly }}{\text { thenumberoftestimages }}
$$

Figure. 10 shows sample retrieval results. A quantitative assessment is performed to evaluate overall performance of the presented retrieval system. Table 1 shows the retrieval performance for normal, moderate and severe classes for macular edema corresponding to severity 0,1 and 2 in Messidor dataset. The average precision and accuracy achieved for the retrieval of top- 5 images are 0.89 and 0.95 whereas for top-10 images it is 0.89 and 0.90 .

Table 2 shows results for test images in two classes, normal versus severe (115 and 96 images respectively). Here the average precision and accuracy for retrieval of top- 5 images are 0.98 and 0.98 whereas for top-10 images are 0.96 and 0.98 .

We observe that the precision for retrieval of "moderate" class images is lower than "normal" and "severe" classes. This is essentially due to two factors. We have taken a 

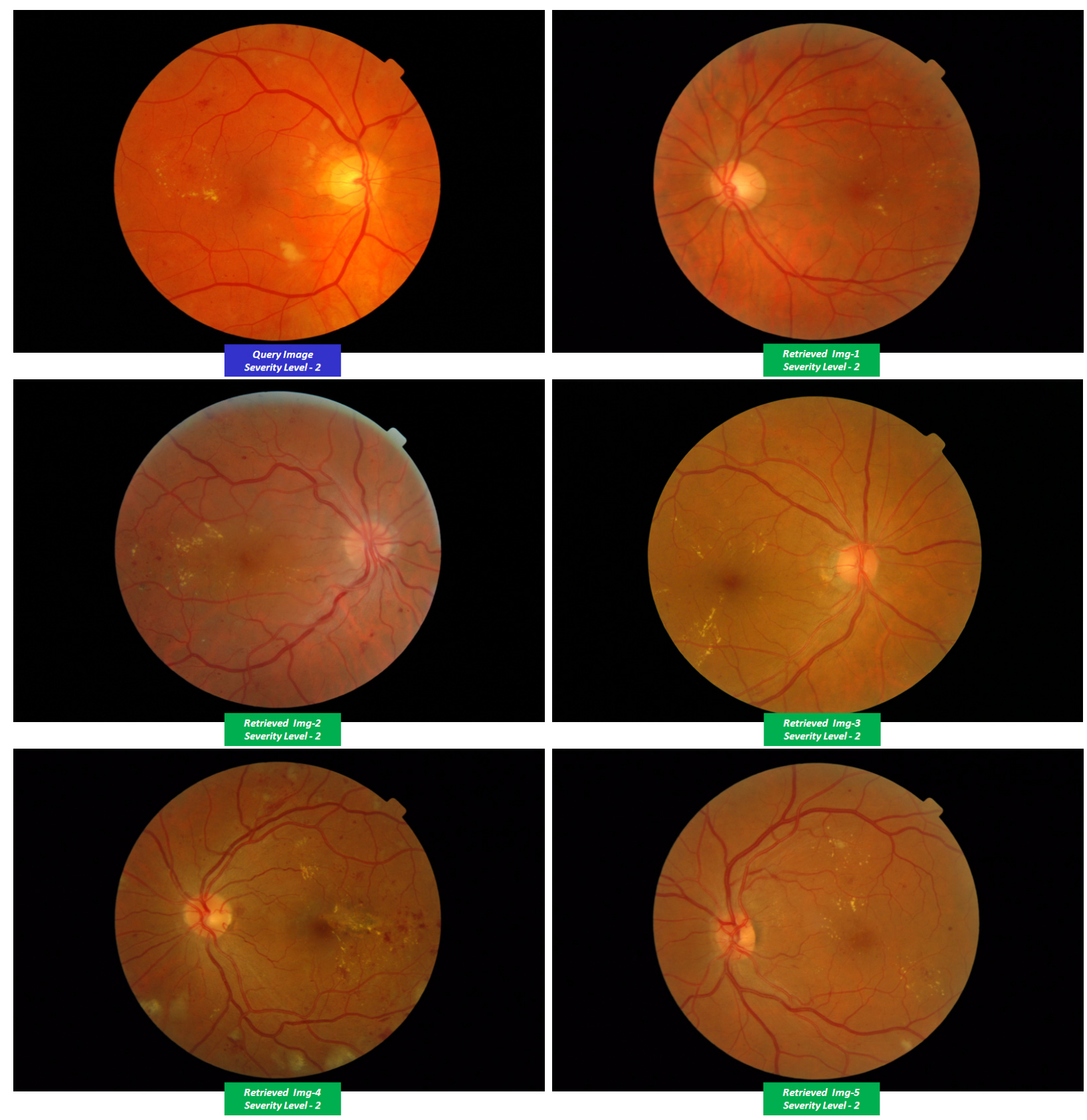

Figure 10: Similar images retrieved (top-5) for the corresponding query image. 
Table 2: Retrieval results for Normal and Severe classes.

\begin{tabular}{|c|c|c|c|}
\hline $\begin{array}{l}\text { Images } \\
\text { Retrieved }\end{array}$ & State & Precision & Accuracy \\
\hline \multirow{3}{*}{5} & Normal & 0.97 & 0.97 \\
\hline & Severe & 0.98 & 0.99 \\
\hline & $A v g$ & 0.98 & 0.98 \\
\hline \multirow{3}{*}{10} & $\overline{\text { Normal }}$ & 0.96 & 0.97 \\
\hline & Severe & 0.96 & 0.99 \\
\hline & Avg & 0.96 & 0.98 \\
\hline
\end{tabular}
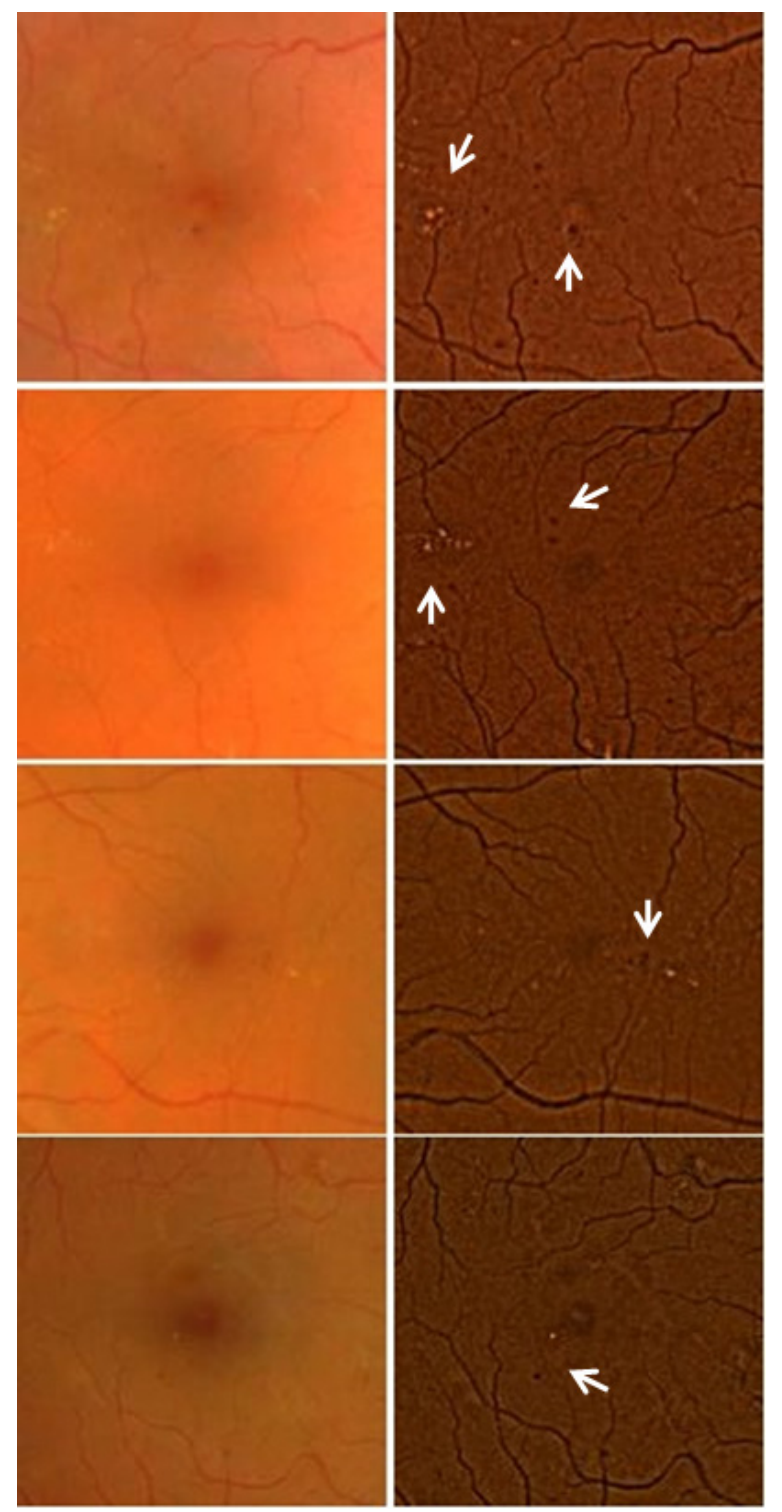

Figure 11: Original and Bias Corrected images marked with severity level "moderate" for macular edema.

maximum radial diameter of 2 papilla (Optic Disk) diameter from the centre of macula as the ROI for analysis. This is considered sufficient as we primarily focus on detection of maculopathy as indicator for macular edema. Few of the images marked as moderate in our dataset have DR lesions outside this range. We have also observed that some of the images marked as moderate have DR lesions very close to the centre of macula which are not visible in the original images (shown in fig 11, left). These lesions are clearly visible after the enhancement step (fig. 11, right). Our system hence assigns a severity level of 2 to these images. The precision for moderate class also suffers because of the fewer number of images pertaining to that class in the dataset.

\section{CONCLUSION}

In this paper, we have described an image retrieval based methodology for achieving high throughput in automated disease-based retrieval and automated diagnosis of macular edema in color fundus images. We have built a disease-based image repository and introduced a new technique for severity level-based retrieval using symmetry. This was tested on an independent dataset showing variability in illumination, pigmentation and resolution of images. The results show that the proposed method is robust and achieves an overall precision of 0.89 and accuracy of 0.95 . This demonstrates the potential of CBIR-based approach for developing a diagnostic aid to readers.

Future work in this area will include integration of automated macula detection to make the entire system automated. Extending the proposed framework to the analysis of the entire retina is possible and this will be useful in determining the severity level of DR.

\section{REFERENCES}

[1] S. T. Acton, P. Soliz, S. R. Russell, and M. S. Pattichis. Content based image retrieval: The foundation for future case-based and evidence-based ophthalmology. In ICME, pages 541-544, 2008.

[2] C. Agurto, V. Murray, E. Barriga, S. Murillo, M. Pattichis, H. Davis, S. Russell, M. Abramoff, and P. Soliz. Multiscale am-fm methods for diabetic retinopathy lesion detection. IEEE Trans Med Imaging, 29(2):502-512, 2010.

[3] M. N. Ahmed, S. M. Yamany, N. Mohamed, A. A. Farag, and T. Moriarty. A modified fuzzy c-means algorithm for bias field estimation and segmentation of mri data. IEEE Trans Med Imaging, 21(3):193-199, 2002.

[4] C. M. Bishop. Neural Networks for Pattern Recognition. Oxford University Press, 1 edition, 1996.

[5] J. Cuadros and G. Bresnick. EyePACS: An Adaptable Telemedicine System for Diabetic Retinopathy Screening. Journal of Diabetes Science and Technology, 3(3):509-516, 2009.

[6] S. Garg, J. Sivaswamy, and S. Chandra. Unsupervised curvature-based retinal vessel segmentation. In Proc. ISBI, pages 344-347, 2007.

[7] A. Gupta, S. Moexxi, A. Taylor, S. Chatterjee, R. Jain, M. Goldbaum, and S. Burgess. Content-based retrieval of ophthamological images. In Proc. ICIP, pages 703-706, 1996.

[8] http://messidor.crihan.fr/index en.php. MESSIDOR, 2004.

[9] R. Hu, S. Ruger, D. Song, H. Liu, and Z. Huang. 
Dissimilarity measures for content-based image retrieval. In Proc. ICME, pages 1365 - 1368, 2008.

[10] T. M. Lehmann, M. O. Güld, C. Thies, B. Plodowski, D. Keysers, B. Ott, and H. Schubert. Irma content-based image retrieval in medical applications. In MEDINFO, pages 842-846, 2004.

[11] H. Liu, D. Song, S. M. Rüger, R. Hu, and V. S. Uren. Comparing dissimilarity measures for content-based image retrieval. In $A I R S$, pages 44-50, 2008.

[12] A. Nikolaos and C. Island. Macula precise localization using digital retinal angiographies. In Proc. 11th WSEAS International Conference on Computers, pages $601-607,2007$.

[13] G. Quellec, M. Lamard, L. Bekri, G. Cazuguel, B. Cochener, and C. Roux. Multimedia medical case retrieval using decision trees. In Proc. EMBS, pages 4536-4539, 2007.
[14] G. Quellec, M. Lamard, G. Cazuguel, C. Roux, and B. Cochener. Multimodal medical case retrieval using the dezert-smarandache theory. In Proc. EMBS, pages 394-397, 2008.

[15] J. Singh, G. D. Joshi, and J. Sivaswamy. Appearance-based object detection in colour retinal images. In Proc. ICIP, pages 1432 - 1435, 2008.

[16] R. Taylor. Handbook of Retinal Screening in Diabetes. John Wiley and Sons, 1 edition, 2006.

[17] K. W. Tobin, M. Abdelrahman, E. Chaum, V. P. Govindasamy, and T. P. Karnowski. A probabilistic framework for content-based diagnosis of retinal disease. In Proc. EMBS, pages 6743-6746, 2007.

[18] K. W. Tobin, M. D. Abramoff, E. Chaum, L. Giancardo, V. P. Govindasamy, T. P. Karnowski, M. T. Tennant, and S. Swainson. Using a patient image archive to diagnose retinopathy. In Proc. $E M B S$, pages 5441-5444, 2008. 